\title{
Investigating children's immersion in a high-embodied versus low-embodied digital learning game in an authentic educational setting
}

\author{
Yiannis Georgiou $^{1,2}$, Andri Ioannou ${ }^{1,2}$ and Marianna Ioannou ${ }^{2}$ \\ ${ }^{1}$ Research center on Interactive media, Smart systems and Emerging technologies (RISE), \\ Nicosia, Cyprus \\ ${ }^{2}$ Cyprus Interaction Lab, Cyprus University of Technology, Limassol, Cyprus
}

\begin{abstract}
Prior research has supported that game-based learning is dependent on the degree of immersion achieved, namely the degree to which children become cognitively and emotionally engaged with a given educational digital game. With the emergence of embodied digital educational games, researchers have assumed that the affordances of these games for movement-based interaction may heighten even more experienced immersion. However, there is lack of empirical research on the investigation of children's immersive experiences in embodied educational games, warranting this claim. Existing research on immersion is still restricted in highly-controlled laboratory settings and focuses on non-educational embodied games played by mostly young adult populations. Extending prior research in the educational context, this study has investigated children's immersion in a high-embodied digital learning game integrated in an authentic school classroom (Group1=24), in comparison to a low-embodied digital version of the game (Group2=20). Our findings did not support previous hypotheses regarding experienced immersion in high-embodied digital games; post-interventional surveys indicated that there was no difference in most dimensions of experienced immersion. Interviews with a subset of the children ( $n=8$ per condition) resulted in the identification of various (a) media form, (b) media content and (c) context-related factors, which provided plausible explanations about children's experienced immersion in the two conditions. Implications are discussed for supporting immersion in high-embodied educational digital games implemented in authentic educational settings.
\end{abstract}

Keywords: Embodied digital games, Children, Immersion, Educational settings

\section{$1 \quad$ Introduction and theoretical framing}

Immersion, as a gradated and multi-level process of cognitive and emotional involvement in digital educational games is often argued as one of the main driving forces behind children's engagement and learning [1-3]. Importantly, an increasing corpus of empirical studies has supported that heightened levels of immersion in digital educational games can increase students' performance and subsequent learning [4- 
7]. The emergence of embodied digital educational games, which are grounded in motion-based technologies (e.g., Wii, Xbox Kinect, Leap Motion) and integrate gestures or even full-body movement into the act of learning [8] has renewed the interest in the investigation of immersive experiences. In particular, researchers have argued that the motion-based affordances of high-embodied digital games may heighten experienced immersion in comparison to prior digital games, as they allow more natural interactions closer to the task to be achieved, reduce the gamers' physical passivity, and provide an additional channel to the gamers for feeling challenged [9-10].

Despite these arguments, there is lack of empirical research with a clear focus on the investigation of children's experienced immersion in high-embodied educational games, warranting these claims. Learning via embodied educational games is still a nascent field. Existing studies have mostly adopted experimental research for investigating whether embodied educational games may have a positive impact on students' learning or not, or design-based research in order to guide the principled development of embodied educational games [11, 12]. According to Karakostas, Palaigeorgiou and Kompatsiaris [13] research on high-embodied digital educational games is still fragmented, driven by specific technological innovations, and often lacking a clear focus on investigating their affordances and limitations within real classroom settings.

Acknowledging the vital role of immersion in relation to students' learning, it seems crucial to obtain a better understanding of whether and under what circumstances embodied digital educational games can have a positive impact on students' experienced immersion, when integrated in authentic educational settings. Despite this realization, relevant empirical studies on the investigation of experienced immersion is limited to the use of non-educational embodied games with young adults' populations in highly-controlled laboratory settings [14, 15]. Taking into account that experienced immersion depends not only on the media form (high-embodied versus low-embodied interfaces), but also on media content factors, user and context-related factors [16-18], in this study we have extended prior research in the educational context.

This study has investigated children's immersion in a high-embodied version (Kinect-based version) of the "Alien Health" digital game when integrated in an authentic school classroom, in comparison to the low-embodied version (Desktop-based version) of the same game. While prior studies, have investigated the learning affordances of the "Alien Health" game and have provided empirical substantiation supporting its learning efficacy $[8,20]$, the present study is focused on investigating children's immersive experiences around this game. In particular, although the game was designed to instruct about nutrition, as part of this study, we were interested in investigating other aspects of this digital game focusing on the following research questions: (a) Is there a difference in children's experienced immersion between the low- and high-embodied digital educational game conditions? and (b) What were the main factors contributing to experienced immersion in the two conditions, as perceived by the children? 


\section{Methods}

\subsection{Participants}

Forty-four $4^{\text {th }}$ graders (aged 8-9 years old), who were enrolled in a public primary school participated in this study. Children were randomly assigned to the two conditions. Group1 (Kinect-based gaming condition) had 24 children (12 boys, 50\%) and Group2 (Desktop-based gaming condition) had 20 children (11 boys, 45\%). Before the intervention consent forms were obtained by the students' legal guardians.

\subsection{Research design}

This study was grounded in an explanatory sequential design, composed of two sequential phases [19]. During the first phase, we adopted a two-group quasiexperimental design for investigating children's learning and immersion per condition. Next, we proceeded with qualitative data collection phase to deepen our understanding of the factors contributing to the children's immersion in each condition.

\subsection{The digital game}

As part of the teaching intervention we have employed the "Alien Health" digital game, which was designed to teach $4^{\text {th }}-12^{\text {th }}$ grades about nutrition $[8,20]$. According to the backstory of the game children are asked to help an Alien, who is in charge of stopping the collision of an asteroid with the Earth; however, the alien is hungry, and he cannot communicate. Children's mission is to make the right nutritional choices for the alien to make him feel better. To achieve this goal, during the gameplay, children are presented with combinations of food, and are requested to make a choice, within a predefined timeframe, considering a constellation of five nutrients per food. The specific digital learning game was chosen for two reasons. First, the digital game was available in both a low-embodied (desktop-based) and in a high-embodied (Kinect-based) version. Second the digital game was subjected into a feasibility study and findings indicated its acceptability by the children as well as its affordances to improve within-group content knowledge $[6,20]$. Although the game was designed to instruct about nutrition, as part of this study, we were interested in investigating other aspects of this digital game, focusing on children's immersive experiences around the game.

\subsection{The interventions}

Considering the research goals of this study, an 80-minute intervention was developed and implemented in an authentic school classroom for each condition. Children in the low-embodied (desktop) condition were divided in dyads and used the desktop-based version of the digital game; in this version children used the mouse and the keyboard for making a choice and feeding the alien (see Figure 1). On the other hand, in the Kinect-based condition, the children worked in groups of four as, due to the limited 
space of the classroom, we could only host up to six Kinect stations; yet the projection screen was large enough to allow access to all the members of the group (see Figure 2). It should also be noted that while the digital game was based on a single player mode, in both conditions it was contextualized in a collaborative educational activity. In particular, the children in each group took turns for playing; the child(ren) in the rest of the group was/were asked to provide feedback to the player and after each round all the players were gathered together for reporting their food choices on a structured worksheet, discussing the selections made.
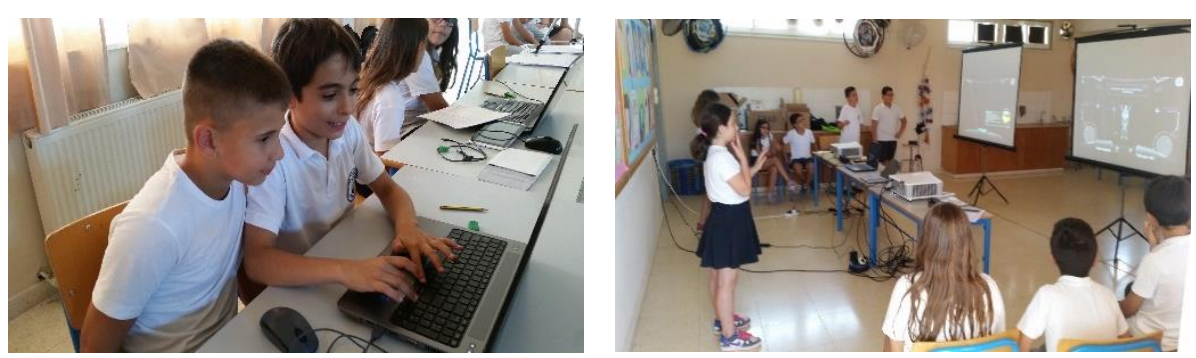

Fig. 1. A group in the low-embodied condition Fig. 2. A group in the high-embodied condition

\subsection{Data collection and analysis}

2.5.1 Baseline data. We collected baseline data using a survey, aiming at creating a profile for the children and establishing the equivalency of the two conditions. The survey had two main parts: Gaming attitudes and Attitudes towards computers. Gaming attitudes was measured using a Likert scale with 11 items, using a five-point Likert scale, derived from the survey of Bonanno and Kommers [21], as this was adapted and validated in the study of Bressler and Bodzin [22]. The Cronbach's a for the adapted instrument was 0.73. Children's attitudes towards computers was assessed using the Computer Attitude Measure for Young Students (CAMYS) [23], which was composed of 12 items, using a five-point Likert scale. The CAMYS is considered a valid instrument and has a documented reliability alpha coefficient of .85 .

2.5.3 Immersion survey. After each implementation, children in each condition completed the Game Immersion Questionnaire (GIQ) which measured their experienced immersion [2]. In particular children were asked to (a) the Engagement scale, which is comprised of three subscales with a total of 9 items (Cronbach's a=0.86): Attraction (4 items, Cronbach's a=0.81), Usability ( 2 items, Cronbach's a=0.73), and Time investment ( 3 items, Cronbach's a $=0.70$ ), (b) the Engrossment scale, which is comprised of two subscales with a total of 7 items (Cronbach's a=0.86): Decreased perceptions (4 items, Cronbach's a=0.79) and Emotional attachment (3 items, Cronbach's $\mathrm{a}=0.79$ ), and (c)the Total Immersion scale, which is comprised of two subscales with a total of 8 items (Cronbach's a=0.92): Presence (4 items, Cronbach's a=0.88) and Empathy (4 items, Cronbach's a=0.87). 
2.5.4 Post-activity interviews. Eight children from each condition participated in semistructured individual interviews, which took place after the intervention. Children were initially asked to report their feelings as these were related to their experienced immersion during the game-based activity (e.g. To what extent did you feel as being within the digital game rather than in the real environment? To what degree did the gaming activity captured all your senses?), and then they were probed to discuss the factors which contributed to their experienced immersion positively or negatively (e.g., What were the main factors contributing to your sense of being [or not] in the digital game? How did these factors contribute [or not] in your gaming experience?).

\section{Data analysis and findings}

\subsection{Setting the baseline}

A Mann-Whitney U test was used to identify any potential differences between children in the two conditions, in terms of their gaming attitudes and attitudes towards computers use. Results showed that there were no statistical differences in the children's gaming attitudes $\left(U_{(42)}=217, z=-.54, p>.05\right)$ and attitudes towards computers $\left(U_{(42)}=203, z=-.87, p>.05\right)$ between the groups

\subsection{Experienced immersion}

A Mann-Whitney U test was used to identify any potential differences in children's experienced immersion (three immersive levels and their dimensions) between the groups. Results showed that there were no statistical differences between the children's Engagement $\left(U_{(42)}=171, z=-1.63, p>.05\right)$, Engrossment $\quad\left(U_{(42)}=233.5, z=-\right.$ $.153, p>.05)$ and Total immersion $\left(U_{(42)}=218, z=-.519, p>.05\right)$ in both conditions. Also, there were no statistically significant differences in the dimensions of the three immersive levels, with one exception. In particular, children in the low-embodied condition perceived the gaming activity as a more user-friendly one compared to the children in the high-embodied condition, and this difference was statistically significant $\left(U_{(42)}=153, z=-2.09, p<.05\right)$.

\subsection{Factors affecting immersion}

All interviews were transcribed and analyzed qualitatively using a top-down thematic analysis approach [24]. The factors identified as having contributed to the children's experienced immersion were classified in two basic themes (Table 1):

(a) Media related factors including media form related factors (referring to the affordances of the gaming platform) and media content related factors (referring to the features of the gaming content) and,

(b) Context related factors (referring to the characteristics of the physical environment and the pedagogical setting in which the game was contextualized). 
These factors are discussed in the following subsections in relation to the two gaming conditions.

Table 1. The factors affecting experienced immersion per condition as reported by the children

\begin{tabular}{|c|c|c|c|}
\hline & & $\begin{array}{l}\text { Condition } 1 \\
\text { Kinect-based } \\
\text { game* }\end{array}$ & $\begin{array}{c}\text { Condition 2 } \\
\text { Desktop-based } \\
\text { game* }\end{array}$ \\
\hline \multirow{8}{*}{$\begin{array}{l}\text { Media-related factors } \\
\text { [Media form] }\end{array}$} & Projection & $\sqrt{ }$ & $\mathrm{x}$ \\
\hline & Interface & $\sqrt{ }$ & $\mathrm{x}$ \\
\hline & Controls & $\mathrm{x}$ & $\sqrt{ }$ \\
\hline & Bodily movement & $\sqrt{ }$ & $\mathrm{n} / \mathrm{a}$ \\
\hline & Embodiment & $\sqrt{ }$ & $\mathrm{n} / \mathrm{a}$ \\
\hline & Single-player mode & $\mathrm{x}$ & $\mathrm{x}$ \\
\hline & Synchronization & $\mathrm{x}$ & $\mathrm{n} / \mathrm{a}$ \\
\hline & Technical bugs & $\mathrm{x}$ & $\mathrm{n} / \mathrm{a}$ \\
\hline \multirow{8}{*}{$\begin{array}{l}\text { Media-related factors } \\
\text { [Media content] }\end{array}$} & Narrative plot & $\sqrt{ }$ & $\sqrt{ }$ \\
\hline & Gaming & $\sqrt{ }$ & $\sqrt{ }$ \\
\hline & Learning nature & $\sqrt{ }$ & $\sqrt{ }$ \\
\hline & Scaffolding & $\sqrt{ }$ & $\sqrt{ }$ \\
\hline & Unrealistic items & $\mathrm{x}$ & $\mathrm{x}$ \\
\hline & Time pressure & $\mathrm{x}$ & $\mathrm{x}$ \\
\hline & Task difficulty & $\mathrm{x}$ & $\mathrm{x}$ \\
\hline & Navigation & $\mathrm{x}$ & $\mathrm{x}$ \\
\hline \multirow[t]{5}{*}{ Context-related factors } & Peer feedback & $\sqrt{ }$ & $\sqrt{ }$ \\
\hline & Collaboration & $\sqrt{ }$ & $\sqrt{ }$ \\
\hline & Waiting time & $\mathrm{x}$ & $\mathrm{n} / \mathrm{a}$ \\
\hline & Classroom arrangement & $\mathrm{x}$ & $\mathrm{n} / \mathrm{a}$ \\
\hline & Classroom noise & $\mathrm{x}$ & $\mathrm{x}$ \\
\hline
\end{tabular}

$*(\sqrt{ })$ indicates the positively evaluated factors, (x) indicates the negatively evaluated factors and (n/a) indicates any factors that were not reported by the children per condition

3.3.1 Media form related factors. According to the children of the high-embodied condition, the large projection (bigger screen providing more heightened sensory stimuli), the interface (with the use of novel technologies), the affordances of the gaming platform for promoting bodily movement (via the players' kinesthetic activity) and embodiment (via the gesture-based interactions) contributed to their experienced immersion. However, the children reported that the controls of the game were rather different from traditional gaming controls (e.g., keyboard, mouse). In particular, according to the children moving the game screens forward with a hand closing action was clunky and tiresome, thus having a negative effect on their experienced immersion. In addition, the children reported that the single-player mode of the game, which transformed more of the group members as spectators, some synchronization issues often presented between player's movements and their belated projection on the screen, as well as some technical bugs (provoked by children's proximity to the Kinect), affected their experienced immersion negatively. On other hand, the children of 
the low-embodied condition evaluated negatively most of the media form related factors. In particular, consistent with the children of the high-embodied condition they disliked the single-player mode of the game. In addition, they reported that the small projection (limited desktop screen) and the interface (traditional desktop-based computer with low graphics) had a negative effect on their experienced immersion. Yet, in contrast to the high-embodied condition, children in the low-embodied condition explained that the familiar desktop computer kept the engaged in the activity.

3.3.2 Media content related factors. Given that the gaming content was similar in both conditions, it is not surprising that children in the high- and low-embodied setting evaluated the media content similarly, in relation to their experienced immersion. In particular, children in both conditions, highlighted the positive impact of the narrative plot, the gaming features (e.g., points and rewards), the integrated scaffolding (available hints on the nutritional value of each food), as well as the learning nature of the game (via an innovative educational approach). On the other hand, children in both conditions highlighted that the unrealistic items (alien creature and, in some cases, alien food), the task difficulty (due to their lack of prior knowledge on the topic), the navigation within the game, and the time pressure (given that the game-based tasks which had to be accomplished in a limited timeframe) had often a negative effect on their experienced immersion.

3.3.3 Context related factors. Focusing on the context related factors, the lowembodied condition appears to be positively linked to the children's experienced immersion. In particular the children highlighted that the collaborative activity which framed the gaming activity, allowed a productive collaboration in their dyads (e.g., dialogue, exchange of views and ideas), while also promoted peer feedback. The only negative evaluated factor was classroom noise, which in some cases could even distract children's attention from the game. In contrast, according to the children in the high-embodied condition, it seems that most of the reported context-related factors were linked to the children's experienced immersion in a negative way. In particular, although children in this condition reported positively with respect to peer feedback, they negatively elaborated on the gaming activity as being framed by an unstructured collaboration with children often fighting over turn-taking and roles in the members of the group. In addition, they explained how the waiting time between turns, often resulted in off-task discussions and behaviors amongst the members of the group. The classroom arrangement which unintentionally allowed access to the projection screens of the other groups served as a distracting factor, as children could borrow solutions or intervene in the discussions and gameplay of other groups. All of these characteristics seemed to result in high levels of classroom noise, which was the most frequently reported factor negatively linked to children's experienced immersion in the high-embodied condition. 


\section{Discussion and implications}

With the emergence of embodied digital educational games, researchers have assumed that the affordances of these games for movement-based interaction may heighten experienced immersion [25]. However, there is lack of empirical research on the investigation of children's immersive experiences in embodied educational games, warranting this claim. Existing research on immersion is still restricted in highlycontrolled laboratory settings and focuses on non-educational embodied games played by mostly young adult populations. Extending prior research in the educational context, this study has investigated children's immersion in a high-embodied digital learning game integrated in an authentic school classroom, in comparison to a lowembodied digital version of the game. Our findings are opposed to the findings of existing research in laboratory settings, which have previously supported the prevalence of high-embodied versus low-embodied games in young adults' experienced immersion $[14,15]$. In particular, the analysis of post-interventional surveys indicated that there was no difference in most dimensions of experienced immersion with one exception. The children in the low-embodied condition deemed their gaming setting as a more user-friendly one, in comparison to the high-embodied condition.

Subsequent analysis of children's interviews regarding the factors affecting immersion between the two groups, shed more light on our findings about the children's immersive experience per condition. In particular, a set of factors related to the media form characteristics, indicated that despite the affordances of a larger projection, locomotion and embodiment, children in the high-embodied condition also reported on synchronization problems, difficulties in using the Kinect, and subsequent technical bugs. These usability issues seem to have had a detrimental effect on children's experienced immersion and could provide a plausible explanation for the difference in children's perceived usability between the two conditions. Additionally, aligned with the theoretical conceptualization of immersion as a multi-level process of cognitive and emotional involvement, such usability issues could serve as major barriers in children's successful transit via the immersive progression [1-3].

On the other hand, it is not a surprise that we have identified no difference on the media content factors when comparing the high- and low-embodied educational conditions, given that the gaming content was the same in both settings. In this context, while many content characteristics (e.g., narrative, gaming, scaffolding) were positively evaluated by the children, what seemed to have a negative influence on children's experienced immersion were the perceived tasks' difficulty in combination with time pressure and navigation difficulties within the game. Finally, what seemed to differentiate at a major degree children's experienced immersion, were a set of factors related to the context characteristics. In alignment with the study of Anderson and Wall [26], who investigated the integration of a high-embodied digital educational game in an authentic school classroom, we too found, for instance, that collaboration in the high-embodied condition was limited and quite unstructured. The detrimental effect on children's experienced immersion was also reinforced by the classroom arrangement in groups of 4 children, which increased the waiting time between players' turns, and resulted in children's off-tasks behaviors and classroom noise. In contrast, children in the low-embodied condition highlighted that the classroom ar- 
rangement in dyads, resulted in a productive collaboration and peer feedback, while also keeping in lower levels the classroom noise.

Overall, the present study provides empirical evidence supporting that children's heightened levels of immersion during the implementation of high-embodied digital games in authentic educational contexts, should not be taken as a given. In addition, grounded in our findings we could state a set of guidelines for supporting children's immersion in high-embodied digital games implemented in real classroom settings:

1. Develop embodied games that integrate intuitive movements, that resemble movements in real life and are aligned to the users' skills and expectations.

2. Organize a training/demonstration session before the gaming activity to allow the children to familiarize themselves with the gaming controls.

3. Introduce and discuss with the children the learning topic before the gaming activity, as this could reduce the tasks' difficulty embedded in the game.

4. Plan for a smart classroom set-up and arrange for up to four Kinect-based stations (one per classroom side), splitting children's groups among the four sides of the classroom and avoiding inter-group interventions and subsequent distractions.

5. Work with smaller cohorts of children (up to 12 children) and divide them in groups of 2-3 members per Kinect station, rather than bigger groups.

6. Contextualize the game in a scripted collaborative setting, where clear roles, responsibilities, and even turn taking will be clear to all members of the group.

7. Proceed with the development of build-in collaboration features in highembodied learning games, for involving simultaneously all the group members.

8. Develop high-embodied games with improved audio-visual characteristics for overpowering the sensory information derived from the physical world.

9. Develop high-embodied learning games combining the use of headsets and cardboard glasses to improve the audio-visual sensory stimuli provided by the game.

The aforementioned guidelines, require the contribution of people from different disciplines: designers, programmers, educators and researchers, working together in the HCI community with a common goal; in our case the design and enactment of more immersive high-embodied educational games in authentic classroom settings, for advancing children's learning.

\section{Acknowledgements}

This work is part of the project that has received funding from the European Union's Horizon 2020 research and innovation programme under grant agreement No 739578 (RISE-Call:H2020-WIDESPREAD-01-2016-2017-TeamingPhase2) and the government of the Republic of Cyprus through the Directorate General for European Programmes, Coordination and Development. 


\section{References}

1. Brown, E., Cairns, P.: A grounded investigation of game immersion. In CHI'04 extended abstracts on Human factors in computing systems, pp. 1297-1300. ACM, (2004).

2. Cheng, M.-T., She, H.-C., Annetta, L. A.: Game immersion experience: Its hierarchical structure and impact on game-based science learning. Journal of Computer Assisted Learning, 31(3), 232-253 (2015).

3. Georgiou, Y., Kyza, E. A.: The development and validation of the ARI questionnaire: An instrument for measuring immersion in location-based augmented reality settings. International Journal of Human Computer Studies, 98, 24-37 (2017).

4. Cheng, M.-T., Linm Y.-W., She, H.-C., Kuo, P.-C.: Is immersion of any value? Whether, and to what extent, game immersion experience during serious gaming affects science learning. British Journal of Educational Technology, 48, 246-263 (2016).

5. Georgiou, Y., Kyza, E. A.: Relations between student motivation, immersion and learning outcomes in location-based augmented reality settings. Computers in Human Behavior, 89, 173-181 (2018).

6. Georgiou, Y., Kyza, E. A.: A design-based approach to augmented reality location-based activities: Investigating immersion in relation to student learning. In: Loizides, F., Papadopoulos, G., Souleles, N. (eds.) $16^{\text {th }}$ World Conference on Mobile and Contextual Learning 2017, mLearn, Vol.1, International Association for Mobile Learning (IAmLearn), Larnaka (2017).

7. Rowe, J. P., Shores, L. R., Mott, B. W., Lester, J. C.: Integrating learning, problem solving, and engagement in narrative-centered learning environments. International Journal of Artificial Intelligence in Education, 21(1-2), 115-133 (2011).

8. Johnson-Glenberg, M. C., Savio-Ramos, C., Henry, H.: “Alien Health": A nutrition instruction exergame using the Kinect sensor. Games for Health Journal. 3(4), 241-251 (2014).

9. Pasch, M., Bianchi-Berthouze, N., Van Dijk, B., Nijholt, A.: Immersion in movementbased interaction. Lecture Notes of the Institute for Computer Sciences, Social-Informatics and Telecommunications Engineering (Vol. 9 LNICST) (2009).

10. Bianchi-Berthouze, N.: Understanding the role of body movement in player engagement. Human-Computer Interaction. 28(1), 40-75 (2013).

11. Georgiou, Y., Ioannou, A.: Embodied learning in a digital world: A systematic review of empirical research in K-12 education. In: Díaz, P., Ioannou, A., Spector, M., \& Bhagat K.K.(eds.) Learning in a digital world: A multidisciplinary perspective on interactive technologies for formal and informal education. Springer series: Smart Computing and Intelligence. Springer (In press).

12. Sheu, F. R., Chen, N. S.: Taking a signal: A review of gesture-based computing research in education. Computers \& Education, 78, 268-277 (2014).

13. Karakostas, A., Palaigeorgiou, G., Kompatsiaris, Y.: WeMake: A framework for letting students create tangible, embedded and embodied environments for their own STEAM learning. In International Conference on Internet Science, pp. 3-18, Cham, Springer, (2017).

14. Coppi, A. E., Lessiter, J., Freeman, J.: Get in the game: the influence of embodied play on presence and flow in videogames. In: Proceedings of the Interactive Technologies and Games Conference (ITAG), Nottingham, United Kingdom (2014).

15. Lindley, S., Couteur, J.L., Bianchi-Berthouze, N: Stirring up experience through movement in game play: Effects on engagement and social behaviour. Proceedings of the SIGCHI Conference on Human Factors in Computing Systems, pp. 511-514. ACM (2008). 
16. Baños, R. M., Botella, C., Alcañiz, M., Liaño, V., Guerrero, B., Rey, B.: Immersion and emotion: Their impact on the sense of presence. Cyberpsychology and Behavior, (7)6, 734-741 (2004).

17. Bleumers, L., Van Lier, T., Jacobs, A.: Presence and mediated interaction: A means to an end? In Networked Television Adjunct proceedings of Euro ITV 2009, pp. 104-106, (2009).

18. Chung, J., Gardner H.J.: Temporal presence variation in immersive computer games. International Journal of Human-Computer Interaction, 28(8), 511-529 (2012).

19. John W. Creswell, P. Clark, L. Vicci: Designing and conducting mixed methods research. Sage Publications, California (2011).

20. Johnson-Glenberg, M.C., Hekler E.B.L: "Alien Health Game”: An embodied exergame to instruct in nutrition and MyPlate. Games for Health Journal, 2(6), 354-361 (2013).

21. Bonanno, P., Kommers, P.A.M.: Exploring the influence of gender and gaming competence on attitudes towards using instructional games. British Journal of Educational Technology, 39(1), 97-109 (2008).

22. Bressler, D.M., Bodzin, A.M.: A mixed methods assessment of students' flow experiences during a mobile augmented reality science game. Journal of Computer Assisted Learning, 29(6), 505-517 (2013).

23. Teo, T., Noyes, J.: Development and validation of a computer attitude measure for young students (CAMYS). Computers in Human Behavior, 24(6), 2659-2667 (2008).

24. Braun, V., Clarke, V.: Using thematic analysis in psychology. Qualitative Research in Psychology. 3(2), 77-101, (2006).

25. Johnson-Glenberg, M.C., Birchfield, D.A., Tolentino, L., Koziupa, T.: Collaborative embodied learning in mixed reality motion-capture environments: Two science studies. Journal of Educational Psychology, 106(1), 86-104 (2014).

26. Anderson, J. L., Wall, S. D.: Kinecting Physics: Conceptualization of motion through visualization and embodiment. Journal of Science Education and Technology, 25(2), 161-173 (2016). 